\title{
CHEBYSHEV EXPANSIONS FOR MODIFIED STRUVE AND RELATED FUNCTIONS
}

\author{
ALLAN J. MACLEOD
}

\begin{abstract}
We consider the approximation of the modified Struve functions $\mathbf{L}_{0}$ and $\mathbf{L}_{1}$, and the related functions $I_{0}-\mathbf{L}_{0}$ and $I_{1}-\mathbf{L}_{1}$, where $I_{0}, I_{1}$ are modified Bessel functions. Chebyshev expansions are derived to an accuracy of 20D for these functions. By using generalized bilinear and biquadratic maps we optimize the number of coefficients for 20D accuracy.
\end{abstract}

\section{INTRODUCTION}

The modified Struve functions $\mathbf{L}_{n}(x)$ satisfy the equation

$$
x^{2} \mathbf{L}_{n}^{\prime \prime}+x \mathbf{L}_{n}^{\prime}-\left(x^{2}+n^{2}\right) \mathbf{L}_{n}=\frac{4(x / 2)^{n+1}}{\sqrt{\pi} \Gamma\left(n+\frac{1}{2}\right)}
$$

and are clearly closely related to the modified Bessel functions of the first kind, $I_{n}(x)$. The functions $\mathbf{L}_{0}$ and $\mathbf{L}_{1}$ appear in the fluid dynamics of water waves; see, for example, Hirata [5] or Shaw [10]. The functions $I_{0}-\mathbf{L}_{0}$ and $I_{1}-\mathbf{L}_{1}$ appear in surface wave problems, Wehausen and Laitone [11], and in unsteady aerodynamics, Ahmadi and Widnall [2]. Tables of values for $\mathbf{L}_{0}$ and $\mathbf{L}_{1}$ (small $x$ ) and $I_{0}-\mathbf{L}_{0}, I_{1}-\mathbf{L}_{1}$ (large $x$ ) appear in Chapter 12 of Abramowitz and Stegun [1]. Luke [6] gives coefficients for Chebyshev expansions for $\mathbf{L}_{0} \mathbf{L}_{1}$ in the range $0 \leq|x| \leq 8$. The computation of $I_{0}-\mathbf{L}_{0}, I_{1}-\mathbf{L}_{1}$ by separate computation of the $I$ and $\mathbf{L}$ functions leads to severe cancellation problems. Desmarais [4] developed expansions for these functions, but his results are incomplete.

In this paper, we derive Chebyshev expansions for the computation of $\mathbf{L}_{0}$ and $\mathbf{L}_{1}$ for all $x$, and $I_{0}-\mathbf{L}_{0}$ and $I_{1}-\mathbf{L}_{1}$ for $x \geq 0$. The coefficients are derived to an accuracy of $20 \mathrm{D}$, with the number of coefficients minimized by generalized mappings. Test procedures show these values give the required accuracy.

\section{Results FOR $\mathbf{L}_{0}, I_{0}-\mathbf{L}_{0}$}

In this section, we describe in detail the procedures used for the functions of order 0 . The methods are then applied to the order-1 functions, so only the relevant results are given in the next section.

Received by the editor March 19, 1992 and, in revised form, June 24, 1992.

1991 Mathematics Subject Classification. Primary 65D20, 65D15, 33C10.

Key words and phrases. Struve functions, Chebyshev expansions, testing. 
$\mathbf{L}_{0}(x)$ has the power series

$$
\mathbf{L}_{0}(x)=\frac{2 x}{\pi}\left(1+\frac{x^{2}}{9}+\frac{x^{4}}{225}+\cdots\right),
$$

which is convergent for all $x$. Thus, $\mathbf{L}_{0}$ is an odd function, so we need only restrict attention to approximating it for $x \geq 0$, then use $\mathbf{L}_{0}(-|x|)=-\mathbf{L}_{0}(|x|)$. Asymptotically, we have

$$
\mathbf{L}_{0}(x) \sim I_{0}(x)-\frac{1}{\pi} \sum_{k=0}^{\infty}\left(\frac{(-1)^{k} \Gamma\left(k+\frac{1}{2}\right)}{\Gamma\left(\frac{1}{2}-k\right)}\right)\left(\frac{2}{x}\right)^{2 k+1} .
$$

The power series for $\mathbf{I}_{0}$ gives

$$
I_{0}-\mathbf{L}_{0}=1-\frac{2 x}{\pi}+\frac{x^{2}}{4}+\cdots
$$

which is decreasing for $x \geq 0$, the physically significant range.

Equation (2.1) suggests that we approximate $\mathbf{L}_{0}$ as

$$
\mathbf{L}_{0}(x)=x g(x), \quad 0 \leq x \leq a,
$$

where we can expand $g(x)$ in terms of Chebyshev polynomials, and use the fact that $g$ will be even. Schonfelder and Razaz [8] showed, however, that such expansions can give rise to serious error amplification if the function $g$ varies greatly in size, as we have with $\mathbf{L}_{0}$. They recommend extracting an explicit exponential term which will absorb most of the function variation, leaving a more stable function to be expanded.

This idea, together with (2.2) and (2.3), suggests the following set of approximations:

$$
\begin{aligned}
& \mathbf{L}_{0}=\frac{2 x}{\pi} e^{x} g_{1}(x), \quad 0 \leq x \leq P, \\
& I_{0}-\mathbf{L}_{0}=g_{2}(x), \quad 0 \leq x \leq P, \\
& I_{0}-\mathbf{L}_{0}=\frac{2}{\pi x} g_{3}(x), \quad x>P,
\end{aligned}
$$

where values of $\mathbf{L}_{0}$ for $x>P$ can be derived from (2.7) and one of the readily available approximations to $I_{0}$. The functions $g_{1}, g_{2}$, and $g_{3}$ are to be expanded as Chebyshev series. Thus, the intervals $[0, P]$ and $(P, \infty)$ need to be transformed into $[-1,1]$. For $g_{1}$ and $g_{2}$ the simple standard transform is $t=2 x / P-1$, while the nature of (2.2) gives for $g_{3}$ the standard form $t=2 P^{2} / x^{2}-1$.

Scraton [9] and Schonfelder [7] have both shown the advantages of more general bilinear and biquadratic maps, so we consider the forms

$$
t=\frac{a x-b}{x+b}, \quad a=1+2 b / P, b>0
$$




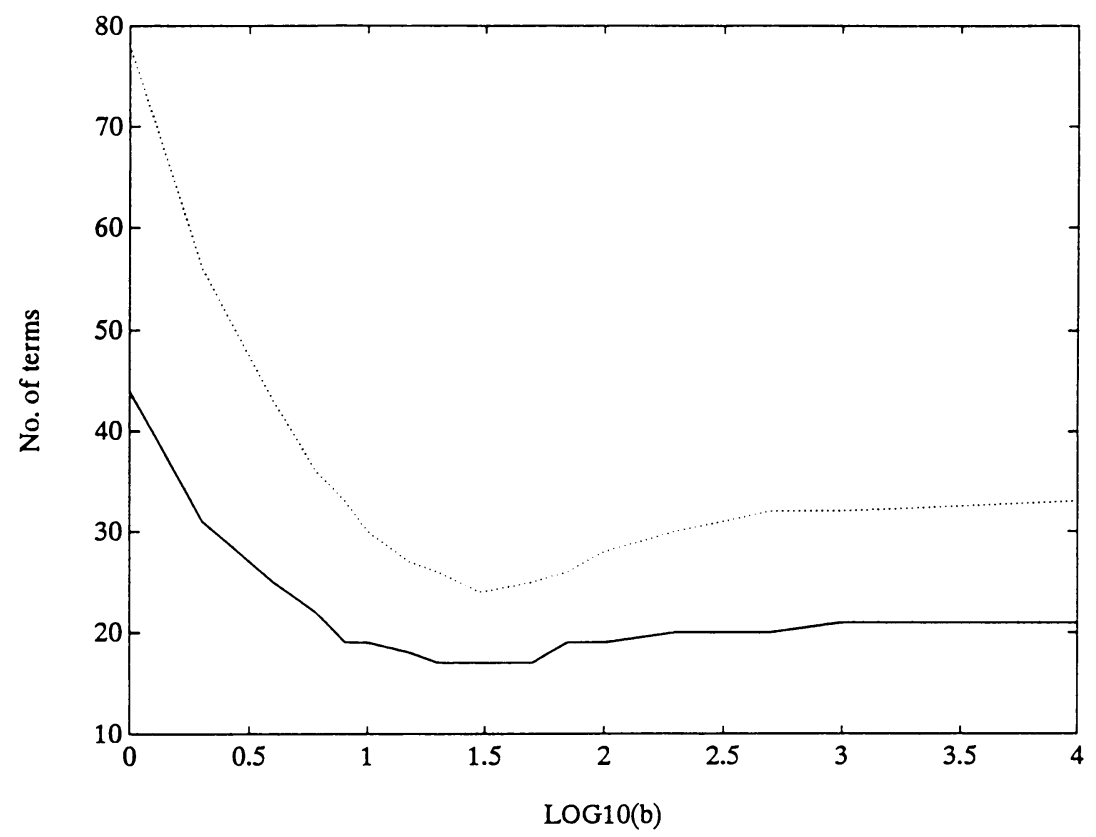

FIGURE 1. Plots for map (2.8)

for $[0, P]$, while for $(P, \infty)$ we consider

$$
t=\frac{x^{2}-c}{d-x^{2}}, \quad d=2 P^{2}-c, c>P^{2} .
$$

To see the effect of such maps, consider $P=4$ and $P=16$, and the expansions for $g_{2}$ and $g_{3}$. The coefficients of the Chebyshev expansion tend to zero fairly quickly, so we can count how many terms are needed before all coefficients are less than $10^{-20}$ in size. Figure 1 shows the number of terms for $g_{2}$, with the solid line representing $P=4$, and the dotted line $P=16$. The standard map is the limit of $(2.8)$ as $b \rightarrow \infty$, giving 21 terms for $P=4$, and 33 terms for $P=16$. The minimum number of terms is 17 for $P=4$, and 24 for $P=16$. Similarly, Figure 2 (next page) shows the same information for $g_{3}$, with, here, the standard map being given by (2.9) with $c=2 P^{2}$. Again, the minimum number of coefficients is below that of the standard map.

We thus have to choose (a) a cutoff value $P$, (b) for this value of $P$, good values for $b$ and $c$, with possibly different $b$-values for $g_{1}$ and $g_{2}$. For each possible combination of $(P, b, c)$-values we generate the Chebyshev coefficients by using Clenshaw's method [3]. This gives an infinite system of linear equations for the coefficients. By assuming that all coefficients beyond a certain point, which is called the zero coefficient cutoff point, are exactly zero, we derive a finite linear system which can be easily solved. To derive values for the parameters, we assumed 101 possibly nonzero coefficients, and performed the arithmetic using quadruple precision on a Prime 6350 (giving about 28 significant decimals).

A large amount of data is obviously generated. The decisions on reasonable values for $P, b, c$ were based on this data. The value $P=16$ was chosen as this gave a simple value, and approximately equal numbers of coefficients 


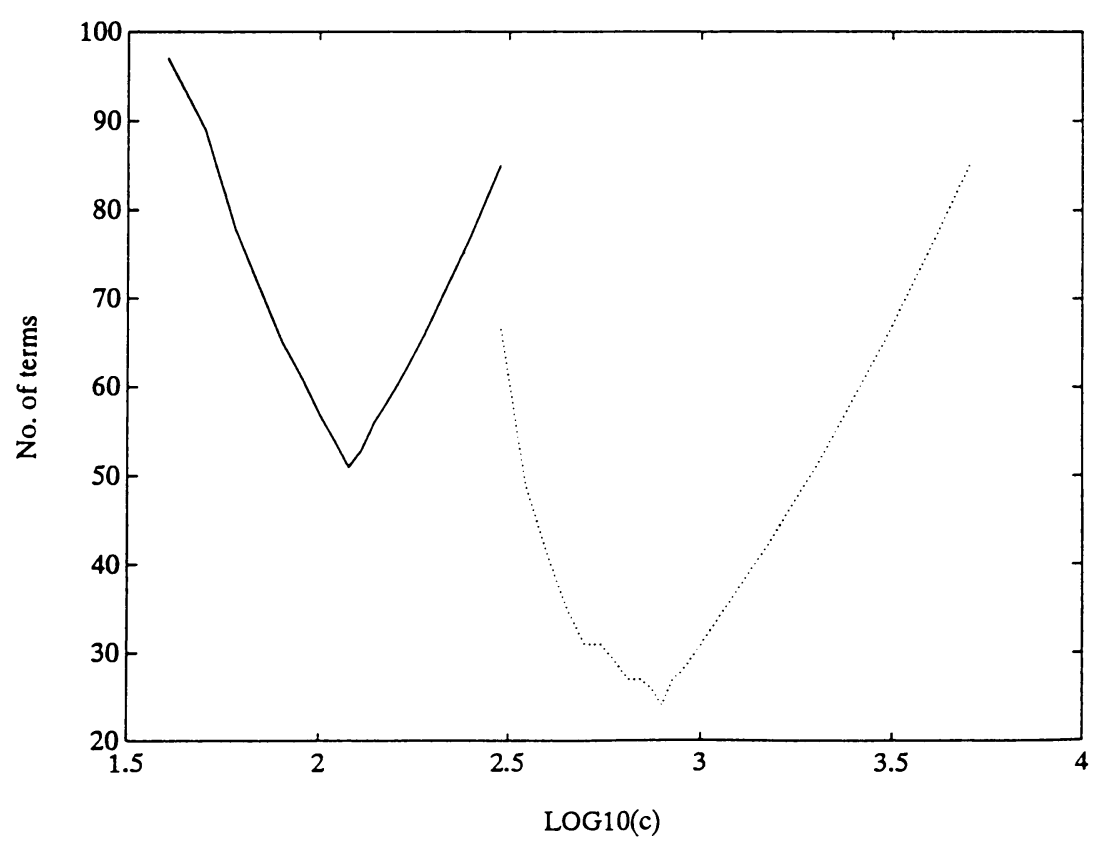

FIGURE 2. Plots for map (2.9)

for $0 \leq x \leq 16$ and $x>16$. With this value of $P$, we varied $b$ and $c$ and found that, for $b$, the minimum number of coefficients occurred over a fairly wide range of values of $b$, while, for $c$, the minimum was more sensitive to values of the parameter. Since we only considered integral $b$ and $c$, the range of $b$ together with (2.8) enabled us to select a value of $b$ giving simple exactly representable transformation coefficients. Integral $c$ means that this also occurs in (2.9). The values chosen led to the following transformations:

$$
\mathbf{L}_{0}: \quad 0 \leq x \leq 16, \quad t=\frac{4 x-24}{x+24},
$$

$$
I_{0}-\mathbf{L}_{0}: \quad 0 \leq x \leq 16, \quad t=\frac{6 x-40}{x+40}
$$

$$
I_{0}-\mathbf{L}_{0}: \quad x>16, \quad t=\frac{800-x^{2}}{288+x^{2}} .
$$

With these transformations fixed, we repeated Clenshaw's method using a multiple-precision floating-point arithmetic package, written by the author. This performed calculations to about 75 significant decimals. The zero coefficient cutoff point was started at 60 , and increased successively by 20 , until the coefficients agreed to $40 \mathrm{D}$. These coefficients $>10^{-20}$ in size were then output, and are given in Tables 1-3. 
TABLE 1. Coefficients for $g_{1}(x)$ for $0 \leq x \leq 16$

$\begin{array}{rccllllr}0 & 0.42127 & 45834 & 99799 & 24863 & \text { E } & 0 \\ 1 & -0.33859 & 53639 & 12206 & 12188 & \text { E } & 0 \\ 2 & 0.21898 & 99481 & 27107 & 16064 & \text { E } & 0 \\ 3 & -0.12349 & 48282 & 07131 & 85712 & \text { E } & 0 \\ 4 & 0.62142 & 09793 & 86695 & 8440 & \text { E } & -1 \\ 5 & -0.28178 & 06028 & 10954 & 7545 & \text { E } & -1 \\ 6 & 0.11574 & 19676 & 63809 & 1209 & \text { E } & -1 \\ 7 & -0.43165 & 85743 & 06921 & 179 & \text { E } & -2 \\ 8 & 0.14614 & 23499 & 07298 & 329 & \text { E } & -2 \\ 9 & -0.44794 & 21180 & 54614 & 78 & \text { E } & -3 \\ 10 & 0.12364 & 74610 & 59437 & 61 & \text { E } & -3 \\ 11 & -0.30490 & 28334 & 79704 & 4 & & \text { E } & -4 \\ 12 & 0.66394 & 14015 & 21146 & & \text { E } & -5 \\ 13 & -0.12553 & 83577 & 03889 & & \text { E } & -5 \\ 14 & 0.20073 & 44645 & 1228 & & \text { E } & -6 \\ 15 & -0.25882 & 60170 & 637 & & \text { E } & -7 \\ 16 & 0.24114 & 37427 & 58 & & \text { E } & -8 \\ 17 & -0.10159 & 67435 & 2 & & \text { E } & -9 \\ 18 & -0.12024 & 30736 & & & \text { E }-10 \\ 19 & 0.26290 & 6137 & & & \text { E }-11 \\ 20 & -0.15313 & 190 & & & \text { E }-12 \\ 21 & -0.15747 & 60 & & & \text { E }-13 \\ 22 & 0.31563 & 5 & & & \text { E }-14 \\ 23 & -0.4096 & & & & \text { E } & -16 \\ 24 & -0.3620 & & & & \text { E }-16 \\ 25 & 0.239 & & & & \text { E } & -17 \\ 26 & 0.36 & & & & \text { E }-18 \\ 27 & -0.4 & & & & \text { E } & -19\end{array}$


TABLE 2. Coefficients for $g_{2}(x)$ for $0 \leq x \leq 16$
$\begin{array}{lllllll}0 & 0.52468 & 73679 & 14855 & 99138 & \mathrm{E} & 0\end{array}$
$\begin{array}{lllllll}1 & -0.35612 & 46049 & 96505 & 86196 & \mathrm{E} & 0\end{array}$
$\begin{array}{lllllll}2 & 0.20487 & 20286 & 40099 & 27687 & \text { E } & 0\end{array}$
$\begin{array}{llllllll}3 & -0.10418 & 64052 & 04026 & 93629 & \mathrm{E} & 0\end{array}$
$\begin{array}{llllllll}4 & 0.46342 & 11095 & 54842 & 9228 & \mathrm{E} & -1\end{array}$
$\begin{array}{llllllll}5 & -0.17905 & 87192 & 40349 & 8630 & \text { E } & -1\end{array}$
$\begin{array}{lllllll}6 & 0.59796 & 86954 & 81143 & 177 & \text { E } & -2\end{array}$
$\begin{array}{lllllll}7 & 0.17177 & 75476 & 93565 & 429 & \text { E } & -2\end{array}$
$\begin{array}{lllllll}8 & 0.42204 & 65446 & 91714 & 22 & \text { E } & -3\end{array}$
$\begin{array}{llllllll}9 & -0.87961 & 78522 & 09412 & 5 & & \text { E } & -4\end{array}$
$\begin{array}{llllllll}10 & 0.15354 & 34234 & 86922 & 3 & & \text { E } & -4\end{array}$
$\begin{array}{llllll}11 & -0.21978 & 07695 & 84743 & \text { E } & -5\end{array}$
$\begin{array}{lllllll}12 & -0.24820 & 68393 & 6666 & \text { E } & -6\end{array}$
$\begin{array}{lllllll}13 & -0.20327 & 06035 & 607 & \text { E } & -7\end{array}$
$\begin{array}{lllllll}14 & 0.90984 & 19842 & 1 & \text { E } & -9\end{array}$
$\begin{array}{llll}15 & 0.25617 & 93929 & \text { E }-10\end{array}$
$\begin{array}{llll}16 & -0.71060 & 9790 & \text { E }\end{array}-11$
$\begin{array}{llll}17 & 0.32716 & 960 & \mathrm{E}-12\end{array}$
$\begin{array}{llll}18 & 0.23002 & 15 & \mathrm{E}-13\end{array}$
$\begin{array}{llll}19 & -0.29210 & 9 & \mathrm{E}-14\end{array}$
$\begin{array}{lll}20 & -0.3566 & \text { E }\end{array}-16$
$\begin{array}{llll}21 & 0.1832 & \mathrm{E} & -16\end{array}$
$\begin{array}{lll}22 & -0.10 & \mathrm{E}-18\end{array}$
$\begin{array}{lll}23 & -0.11 & \text { E }-18\end{array}$ 
TABLE 3. Coefficients for $g_{3}(x)$ for $x>16$

\begin{tabular}{|c|c|c|c|c|c|c|}
\hline 0 & 2.00326 & 51024 & 11606 & 43125 & $\mathrm{E}$ & 0 \\
\hline 1 & 0.19520 & 68515 & 76492 & 081 & $E$ & -2 \\
\hline 2 & 0.38239 & 52356 & 99083 & 28 & $\mathrm{E}$ & -3 \\
\hline 3 & 0.75342 & 80817 & 05443 & 6 & $E$ & -4 \\
\hline 4 & 0.14959 & 57655 & 89707 & 8 & $\mathrm{E}$ & -4 \\
\hline 5 & 0.29994 & 05312 & 10557 & & $\mathrm{E}$ & -5 \\
\hline 6 & 0.60769 & 60482 & 2459 & & $E$ & -6 \\
\hline 7 & 0.12399 & 49554 & 4506 & & $\mathrm{E}$ & -6 \\
\hline 8 & 0.25232 & 62552 & 649 & & $\mathrm{E}$ & -7 \\
\hline 9 & 0.50463 & 48573 & 32 & & $E$ & -8 \\
\hline 10 & 0.97913 & 23623 & 0 & & $\mathrm{E}$ & -9 \\
\hline 11 & 0.18389 & 11524 & 1 & & $\mathrm{E}$ & -9 \\
\hline 12 & 0.33763 & 09278 & & & $E$ & -10 \\
\hline 13 & 0.61117 & 9703 & & & $\mathrm{E}$ & -11 \\
\hline 14 & 0.10847 & 2972 & & & $E$ & -11 \\
\hline 15 & 0.18861 & 271 & & & $\mathrm{E}$ & -12 \\
\hline 16 & 0.32803 & 45 & & & $E$ & -13 \\
\hline 17 & 0.56564 & 7 & & & $E$ & -14 \\
\hline 18 & 0.93300 & & & & $E$ & -15 \\
\hline 19 & 0.15881 & & & & $E$ & -15 \\
\hline 20 & 0.2791 & & & & $\mathrm{E}$ & -16 \\
\hline 21 & 0.389 & & & & $E$ & -17 \\
\hline 22 & 0.70 & & & & $E$ & -18 \\
\hline 23 & 0.16 & & & & $\mathrm{E}$ & -18 \\
\hline
\end{tabular}




\section{RESUlts FOR $\mathbf{L}_{1}, I_{1}-\mathbf{L}_{1}$}

The relevant expansions are

$$
\begin{aligned}
\mathbf{L}_{1}(x) & =\frac{2 x^{2}}{\pi}\left[\frac{1}{3}+\frac{x^{2}}{45}+\frac{x^{4}}{1575}+\cdots\right], \\
I_{1}(x) & =\frac{x}{2}+\frac{x^{3}}{16}+\frac{x^{5}}{384}+\cdots, \\
I_{1}-\mathbf{L}_{1} & \sim \frac{2}{\pi}\left[1-\frac{1}{x^{2}}-\frac{3}{x^{4}}-\frac{45}{x^{6}}-\cdots\right] .
\end{aligned}
$$

These expansions give rise to the approximating forms

$$
\begin{aligned}
\mathbf{L}_{1} & =\frac{2 x^{2}}{3 \pi} e^{x} g_{4}(x), \quad 0 \leq x \leq P, \\
I_{1}-\mathbf{L}_{1} & =\frac{x}{2} g_{5}(x), \quad 0 \leq x \leq P, \\
I_{1}-\mathbf{L}_{1} & =\frac{2}{\pi} g_{6}(x), \quad x>P .
\end{aligned}
$$

The functions $g_{4}, g_{5}, g_{6}$ are expanded in Chebyshev polynomials. Exactly the same investigation procedure as in $\S 2$ showed that $P=16$ was again a reasonable choice, and the transformations could be taken from order 0 to the corresponding order 1 functions. Thus $g_{4}, g_{5}$, and $g_{6}$, use respectively (2.10), (2.11), and (2.12).

The 20D coefficients produced by the multiple-precision software are given in Tables 4-6.

\section{TESTING}

The main test used was to write a Fortran program to evaluate $g_{1}$ to $g_{6}$ for various values of $x$ (using quadruple precision), and to compare the results with values calculated in other ways.

For $0 \leq x \leq 16$, the various power series for $\mathbf{L}_{0}, \mathbf{L}_{1}, I_{0}-\mathbf{L}_{0}, I_{1}-\mathbf{L}_{1}$ were used to generate comparison values. In each unit interval, 1000 random values were generated and in all cases the maximum absolute error was less than $.5 \times 10^{-19}$.

For $g_{3}$ and $g_{6}$ in the range $x>16$ we experienced more problems in testing. For $x$ close to 16 the power series still gives sufficient accuracy. For large $x$, the asymptotic series was transformed into a continued fraction by the $q-d$ method, and gave sufficient accuracy. There was, however, an interval from about $x=25$ to $x=50$ where we were unable to generate sufficiently accurate comparison values from either the power series or the continued fraction.

To get around this, we had to generate the comparison values from the power series using the multiple-precision package. The quadruple-precision argument $x$ was transformed to multiple-precision form exactly by equivalencing it to eight 2-byte integers and decomposing these to give the exact binary representation. By using a base of $2^{12}$ in the package we preserve exactness. The results of the multiple-precision power series were then transformed back to quadruple-precision form. These results verified 20D absolute accuracy for $g_{3}$ and $g_{6}$. 
TABLE 4. Coefficients for $g_{4}(x)$ for $0 \leq x \leq 16$

$\begin{array}{rrrlllll}0 & 0.38996 & 02735 & 12295 & 38208 & \text { E } & 0 \\ 1 & -0.33658 & 09610 & 19757 & 49366 & \text { E } & 0 \\ 2 & 0.23012 & 46791 & 25016 & 45616 & \text { E } & 0 \\ 3 & -0.13121 & 59400 & 79608 & 32327 & \text { E } & 0 \\ 4 & 0.64259 & 22289 & 91284 & 6518 & \text { E } & -1 \\ 5 & -0.27500 & 32950 & 61663 & 5833 & \text { E } & -1 \\ 6 & 0.10402 & 34148 & 63720 & 8871 & \text { E } & -1 \\ 7 & -0.35053 & 22949 & 36388 & 080 & \text { E } & -2 \\ 8 & 0.10574 & 84984 & 21439 & 717 & \text { E } & -2 \\ 9 & -0.28609 & 42640 & 36665 & 58 & \text { E } & -3 \\ 10 & 0.69257 & 08785 & 94220 & 8 & & \text { E } & -4 \\ 11 & -0.14896 & 93951 & 12271 & 7 & & \text { E } & -4 \\ 12 & 0.28103 & 55825 & 97128 & & \text { E } & -5 \\ 13 & -0.45503 & 87929 & 7776 & & \text { E } & -6 \\ 14 & 0.60901 & 71561 & 770 & & \text { E } & -7 \\ 15 & -0.62354 & 37248 & 08 & & \text { E } & -8 \\ 16 & 0.38430 & 01206 & 7 & & \text { E } & -9 \\ 17 & 0.79054 & 3916 & & & \text { E }-11 \\ 18 & -0.48982 & 4083 & & & \text { E }-11 \\ 19 & 0.46356 & 884 & & & \text { E }-12 \\ 20 & 0.68420 & 5 & & & \text { E }-14 \\ 21 & -0.56974 & 8 & & & \text { E }-14 \\ 22 & 0.35324 & & & & \text { E }-15 \\ 23 & 0.4244 & & & & \text { E } & -16 \\ 24 & -0.644 & & & & \text { E }-17 \\ 25 & -0.21 & & & & \text { E } & -18 \\ 26 & 0.9 & & & & \text { E }-19\end{array}$


TABLE 5. Coefficients for $g_{5}(x)$ for $0 \leq x \leq 16$

$\begin{array}{rrrlllll}0 & 0.67536 & 36906 & 23505 & 76137 & \text { E } & 0 \\ 1 & -0.38134 & 97109 & 72665 & 59040 & \text { E } & 0 \\ 2 & 0.17452 & 17077 & 51339 & 43559 & \text { E } & 0 \\ 3 & -0.70621 & 05887 & 23502 & 5061 & \text { E } & -1 \\ 4 & 0.25173 & 41413 & 55880 & 3702 & \text { E } & -1 \\ 5 & -0.78709 & 85616 & 06423 & 321 & \text { E } & -2 \\ 6 & 0.21481 & 43686 & 51922 & 006 & \text { E } & -2 \\ 7 & -0.50862 & 19971 & 79062 & 36 & & \text { E } & -3 \\ 8 & 0.10362 & 60828 & 04423 & 30 & \text { E } & -3 \\ 9 & -0.17954 & 47212 & 05724 & 7 & & \text { E } & -4 \\ 10 & 0.25978 & 82745 & 15414 & & \text { E } & -5 \\ 11 & -0.30442 & 40632 & 4667 & & & \text { E } & -6 \\ 12 & 0.27202 & 39894 & 766 & & \text { E } & -7 \\ 13 & -0.15812 & 61441 & 90 & & & \text { E } & -8 \\ 14 & 0.18162 & 09172 & & & \text { E }-10 \\ 15 & 0.64796 & 7659 & & & \text { E }-11 \\ 16 & -0.54113 & 290 & & & \text { E }-12 \\ 17 & -0.30831 & 1 & & & & \text { E }-14 \\ 18 & 0.30563 & 8 & & & \text { E }-14 \\ 19 & -0.9717 & & & & \text { E }-16 \\ 20 & -0.1422 & & & & \text { E }-16 \\ 21 & 0.84 & & & & \text { E }-18 \\ 22 & 0.7 & & & & \text { E }-19 \\ 23 & -0.1 & & & & \text { E }-19\end{array}$


TABLE 6. Coefficients for $g_{6}(x)$ for $x>16$

\begin{tabular}{|c|c|c|c|c|c|c|}
\hline 0 & 1.99679 & 36189 & 67891 & 36501 & $E$ & 0 \\
\hline 1 & -0.19066 & 32614 & 09686 & 132 & E & -2 \\
\hline 2 & -0.36094 & 62241 & 01744 & 81 & E & -3 \\
\hline 3 & -0.68418 & 47304 & 59982 & 0 & E & -4 \\
\hline 4 & -0.12990 & 08228 & 50942 & 6 & $\mathrm{E}$ & -4 \\
\hline 5 & -0.24715 & 21887 & 05765 & & $\mathrm{E}$ & -5 \\
\hline 6 & -0.47147 & 83969 & 1972 & & E & -6 \\
\hline 7 & -0.90208 & 19982 & 592 & & E & -7 \\
\hline 8 & -0.17304 & 58637 & 504 & & $\mathrm{E}$ & -7 \\
\hline 9 & -0.33232 & 36701 & 59 & & $\mathrm{E}$ & -8 \\
\hline 10 & -0.63736 & 42173 & 5 & & E & -9 \\
\hline 11 & -0.12180 & 23975 & 6 & & $\mathrm{E}$ & -9 \\
\hline 12 & -0.23173 & 46832 & & & $\mathrm{E}$ & -10 \\
\hline 13 & -0.43906 & 8833 & & & $\mathrm{E}$ & -11 \\
\hline 14 & -0.82847 & 110 & & & $\mathrm{E}$ & -12 \\
\hline 15 & -0.15562 & 249 & & & $\mathrm{E}$ & -12 \\
\hline 16 & -0.29131 & 12 & & & $\mathrm{E}$ & -13 \\
\hline 17 & -0.54396 & 5 & & & $\mathrm{E}$ & -14 \\
\hline 18 & -0.10117 & 7 & & & $\mathrm{E}$ & -14 \\
\hline 19 & -0.18767 & & & & $\mathrm{E}$ & -15 \\
\hline 20 & -0.3484 & & & & $\mathrm{E}$ & -16 \\
\hline 21 & -0.643 & & & & $\mathrm{E}$ & -17 \\
\hline 22 & -0.118 & & & & $\mathrm{E}$ & -17 \\
\hline 23 & -0.22 & & & & $\mathrm{E}$ & -18 \\
\hline 24 & -0.4 & & & & $\mathrm{E}$ & -19 \\
\hline 25 & -0.1 & & & & $\mathrm{E}$ & -19 \\
\hline
\end{tabular}


TABLE 7. Coefficients for relationship (4.6)

\begin{tabular}{ccc}
\hline$j$ & $r_{i, j}$ & $s_{i, j}$ \\
\hline-3 & $(3-i) / 8 i$ & - \\
-2 & $(9 i-18) / 2 i$ & $1400 / i$ \\
-1 & $435(1-i) / 8 i$ & $2800 / i$ \\
0 & 225 & 0 \\
1 & $-435(1+i) / 8 i$ & $-2800 / i$ \\
2 & $9(i+2) / 21$ & $-1400 / i$ \\
3 & $-(3+i) / 8 i$ & - \\
\hline
\end{tabular}

A completely different test can be performed using the relationships between the functions of different orders and properties of Chebyshev polynomials. We illustrate the method for $I_{0}-\mathbf{L}_{0}$ and $I_{1}-\mathbf{L}_{1}$ in the range $0 \leq x \leq 16$.

We have $I_{0}^{\prime}=I_{1}$ and $\mathbf{L}_{0}^{\prime}=\mathbf{L}_{1}+\frac{2}{\pi}$ so

$$
\left(I_{0}-\mathbf{L}_{0}\right)^{\prime}=\left(I_{1}-\mathbf{L}_{1}\right)-2 / \pi
$$

giving

$$
g_{2}^{\prime}=-\frac{2}{\pi}+\frac{x}{2} g_{5}
$$

Since $t=(6 x-40) /(x+40)$, standard algebra gives

$$
(6-t)^{3} \frac{d g_{2}}{d t}=5600(1+t) g_{5}-\frac{560}{\pi}(6-t) .
$$

Integrating gives

$$
\begin{aligned}
(216 & \left.-108 t+18 t^{2}-t^{3}\right) g_{2}+\int\left(108-36 t+3 t^{2}\right) g_{2} \\
& =5600 \int(1+t) g_{5}+\frac{280}{\pi} t^{2}-\frac{3360}{\pi} t .
\end{aligned}
$$

Let

$$
g_{2}=\sum_{i=0}^{\infty} c_{i} T_{i}(t), \quad g_{5}=\sum_{i=0}^{\infty} 57 d_{i} T_{i}(t)
$$

then, using standard Chebyshev relationships, we derive

$$
\sum_{j=-3}^{3} r_{i j} c_{i+j}=\sum_{j=-2}^{+2} s_{i j} d_{i+j}, \quad i=3,4,5, \ldots,
$$

where the coefficients $r_{i j}(j=-3, \ldots, 3)$ and $s_{i j}(j=-2, \ldots, 2)$ are given in Table 7. Applying these relationships to the coefficients, we get agreement to within acceptable rounding error. Similar techniques can be applied to relate 
the coefficients of $\mathbf{L}_{0}$ and $\mathbf{L}_{1}$, and the asymptotic forms of $I_{0}-\mathbf{L}_{0}$ and $I_{1}-\mathbf{L}_{1}$. Again acceptable agreement is found.

This mixture of tests leads us to accept the given coefficients as accurate.

\section{BIBLIOGRAPHY}

1. M. Abramowitz and I. A. Stegun, Handbook of mathematical functions, Dover, New York, 1965.

2. A. R. Ahmadi and S. E. Widnall, Unsteady lifting-line theory as a singular-perturbation problem, J. Fluid Mech. 153 (1985), 59-81.

3. C. W. Clenshaw, Chebyshev series for mathematical functions, NPL Math Tables 5, Her Majesty's Stationery Office, London, 1962.

4. R. N. Desmarais, Accurate numerical evaluation of modified Struve functions occurring in unsteady aerodynamics, J. Aircraft 16 (1979), 441-447.

5. M. H. Hirata, Flow near the bow of a steadily turning ship, J. Fluid Mech. 71 (1975), 283-291.

6. Y. L. Luke, The special functions and their approximations. Vol. 2, Academic Press, New York, 1968.

7. J. L. Schonfelder, Chebyshev expansions for the error and related functions, Math. Comp. 32 (1978), 1232-1240.

8. J. L. Schonfelder and M. Razaz, Error control with polynomial approximation, IMA J. Numer. Anal. 1 (1980), 105-114.

9. R. E. Scraton, A method for improving the convergence of Chebyshev series, Comput. J. 13 (1970), 202-203.

10. D. C. Shaw, Perturbational results for diffraction of water waves by nearly vertical barriers, IMA J. App. Math. 34 (1985), 99-117.

11. J. V. Wehausen and E. V. Laitone, Surface waves, Handbuch der Physik, vol. 9, SpringerVerlag, Berlin 1960.

Department of Mathematics and Statistics, University of Paisley, High Street, Paisley, Scotland PA1 2BE

E-mail address: amcl@uk.ac.paisley.cs 